(c) 2011 IEEE. Personal use of this material is permitted. Permission from IEEE must be obtained for all other uses, in any current or future media, including reprinting/republishing this material for advertising or promotional purposes, creating new collective works, for resale or redistribution to servers or lists, or reuse of any copyrighted component of this work in other works. 


\title{
Thermographic Investigation of Osseous Stress Pathology
}

\author{
Daniel TJ. Arthur, Masood Mehmood Khan and Luke C. Barclay
}

\begin{abstract}
The debilitating pathology of stress fracture accounts for $10 \%$ of all athletic injuries[2], with prevalence as high as $20 \%$ in modern military basic training cohorts [3]. Increasing concerns surrounding adverse effects of radiology [5], combined with the $12.5 \%$ contribution of diagnostic imaging to Australian Medicare benefits paid in 2009-10 [6], have prompted the search for alternative/adjunct electronic decision support systems[7]. Within conducive physioanatomic milieu, thermal infrared imaging (TIRI) may feasibly be used to remotely detect and topographically map diagnostically useful signs of suprathreshold thermodynamic pathophysiology. This paper details a three month clinical pilot study into TIRI-based detection of osseous stress pathology in the lower legs of Australian Army basic trainees. A dataset of over 500 TIRI's was amassed. The apparent 'normal' thermal profile of the anterior aspect of the asymptomatic lower leg is topographically defined and validated against current thermophysiological theory [8] via cadaveric dissection.
\end{abstract}

\section{INTRODUCTION}

$\mathrm{T}$ HERMAL infrared imaging (TIRI) employs a focal plane array (FPA) to remotely detect and topographically map thermal emittance. Suprathreshold thermodynamic pathophysiology occurring in conducive pathoanatomic milieu may feasibly manifest TIR 'signs'. Osseous stress pathophysiology features focal thermodynamic mechanisms including hyperemia, and both osteocytic and hyperalgesic nitric oxide production [9]. In physioanatomic context of the anteromedial aspects of the tibiae, as pertinent to epidemiological prevalence [3], these mechanisms occur within a thin superficial volume of thermodynamically determinate tissues [10], unoccluded by thermogenic anatomy or dissipative heat-sinking vasculature [11]. The aim of this study was to elucidate the potential of TIRI in the diagnosis and/or management of osseous stress pathology in the lower

Manuscript received March 19, 2011.

Masood Mehmood Khan is with the Faculty of Science and Engineering, Curtin University of Technology Perth, WA 6102, AUS, phone: +61 89266 9205; e-mail: masood.khan@ curtin.edu.au

D. TJ. Arthur is with Curtin University of Technology Department of Mechanical Engineering, Perth, WA 6102, AUS, phone: +61 89266 2886; email: d.arthur@postgrad.curtin.edu.au

L. C. Barclay is with Curtin University of Technology Department of Medical Imaging and Applied Physics, Perth, WA 6102, AUS, phone: +61 8 9266 2299; e-mail: L.Barclay@ curtin.edu.au

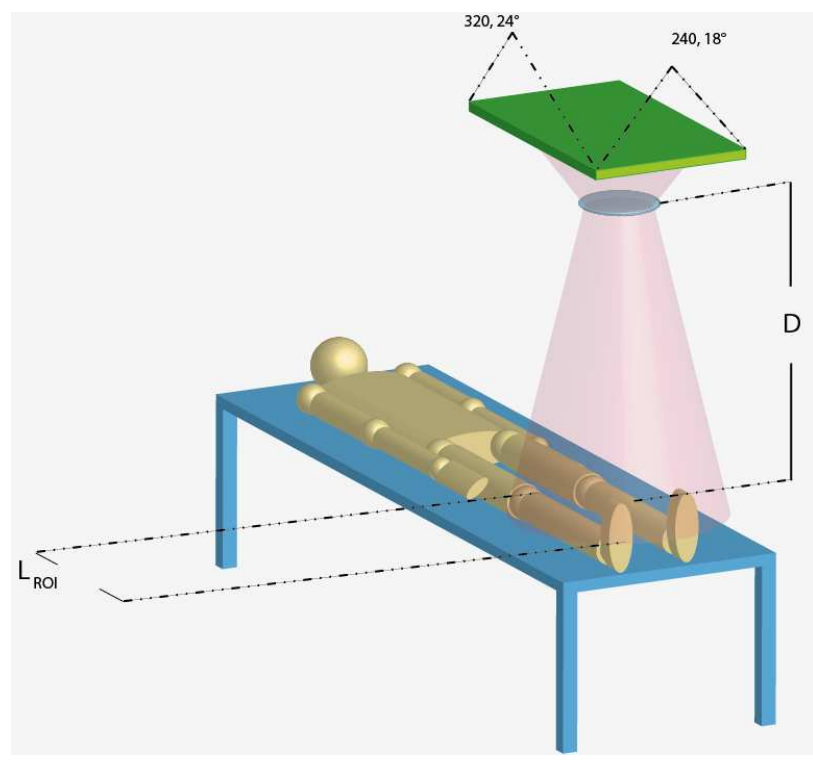

Fig. 1. Clinical image acquisition set-up. $\mathrm{L}_{\mathrm{ROI}}=$ longest dimension of the region of interest corresponding to the popliteal height; $\mathrm{D}=$ lens to skinsurface distance; $320 \times 240$ microbolometric FPA with $24^{\circ} \times 18^{\circ} \Theta_{\mathrm{FOV}}$ shown in green.

limbs, via; topographic definition of the 'normal' asymptomatic thermal profile of the anterior tibial ROI; compilation of a multi-modality symptomatic dataset for validation of TIR signs and development of a pathoanatomically representative bioheat transfer model; and compilation of longitudinal intrasubject TIR datasets to elucidate the potential for TIR detection of subclinical pathology and general pathological staging. In light of the afore-alluded-to feasibility study, and development of objective clinical protocols via functional isolation of the inherent systems' architecture, the authors were granted approval for protocols 592-10 and HR62/2010 by the Australian Defence Force and Curtin University human research ethics committees, respectively.

\section{MATERIALS AND METHODS}

Upon arrival at the Australian Army Recruit Training Centre, three platoons of basic trainees consisting of 56, 48, and 43 recruits were given a comprehensive plain-language study briefing, and allowed 24 hours to anonymously deposit consent forms featuring medical questions pertinent to 
contraindication of TIRI into a sensitively located ballot box. Consenting participants were first thermally imaged during their initial inoculation parade, occurring prior to their first physical training session. Thermal infrared images were acquired prior to vaccine administration to avoid vasoactive artifacts. In absence of data pertinent to optimal conditions for pre-TIRI equilibration, participants remained seated, clad in loose fitting gym shorts and T-shirts, without shoes or socks, for a nominal 20-30 minute period, in a carpeted room with ambient conditions as listed in Table 1.

TABLE I

AMBIENT CLINICAL CONDITIONS

\begin{tabular}{|c|c|c|c|}
\hline Parameter & $\begin{array}{c}\text { Optimal } \\
\text { Thermoneutral } \\
\text { Value* } \\
\end{array}$ & $\begin{array}{l}\text { Experimental } \\
\text { Value }\end{array}$ & $\begin{array}{c}\text { Meter / Reference } \\
\text { for Experimental } \\
\text { Value }\end{array}$ \\
\hline $\begin{array}{l}\text { Ambient Air } \\
\text { Temperature }\end{array}$ & $30^{\circ} \mathrm{C}$ & $28^{\circ} \mathrm{C} \pm 1^{\circ} \mathrm{C}$ & $\begin{array}{l}\text { Vaisala } \\
\text { HUMICAP® } \\
\text { HM34 }\end{array}$ \\
\hline $\begin{array}{l}\text { Relative } \\
\text { Humidity }\end{array}$ & $40 \%$ & $46 \% \pm 3 \%$ & $\begin{array}{l}\text { Vaisala } \\
\text { HUMICAP® } \\
\text { HM34 }\end{array}$ \\
\hline $\begin{array}{l}\text { Emissivity of } \\
\text { surrounding }\end{array}$ & 0.93 & $0.92-0.96$ & [1] \\
\hline
\end{tabular}

\section{Temperature of}

surrounding

$30^{\circ} \mathrm{C}$

Not Controlled

N/A

surfaces

Ambient Air $0.05 \mathrm{~m} / \mathrm{s}$

\section{Minimized}

Velocity

where

practicable but

N/A not monitored

Minimized

Basal Metabolic

Rate

0.8

where

practicable but

not monitored

*Optimal thermoneutral values as calculated via the IESD-Fiala human thermoregulation model used in conjunction with ANSYS CFX indoor environment program [4].

During equilibration each participant's legs were visually photographed to screen potentially contraindicative superficial lesions. Following equilibration, patients lay stationary supine upon an unarticulated/flat LINAK OpenBus ${ }^{\mathrm{TM}}$ hospital bed, for no less than 3 minutes prior to image acquisition. Several $2 \mathrm{~cm}$ thick dark grey matt-finish gym mats were kept in redundancy, equilibrating on rotation to $20^{\circ} \mathrm{C}$ in an anteroom and used as a backing surface between the bed and each participant to prevent residual thermal artifacts. An ad-hoc camera mounting system was constructed to steadily maintain the lens to skin-surface distance ('D' Fig. 1), with the optical axis normal to the patient bed. In absence of data concerning optimal ambient conditions for image capture, five wallmounted Daikin FVXS71H ducted inverter units were used to maintain conditions as close to thermoneutral (Table 1) as practicable. Pending release of the IEC TC/SC/WG5 specifications for medical grade thermal infrared cameras [12], a FLIR ThermoVision ${ }^{\mathrm{TM}}$ A40V thermal infrared camera featuring a 7.5 to $13.0 \mu \mathrm{m}$ uncooled bolometric FPA and thermal sensitivity of $0.08^{\circ} \mathrm{C}$ was used in the configuration shown in Fig.1. The longest dimension of the region of interest
$\left(\mathrm{L}_{\mathrm{roi}}\right)$ was anticipated to be $\leq 515 \mathrm{~mm}$ in accordance with $95^{\text {th }}$ percentile 'popliteal height' as defined in ISO/TR 72502:2010 [13]. Given the A40V's 320x240 FPA with $\Theta_{\mathrm{FOV}}$ of $24^{\circ} \times 18^{\circ}$ [14], the lens to skin-surface distance ('D' Fig. 1) was set to $1.30 \mathrm{~m}$, providing a $553 \mathrm{~mm} \times 412 \mathrm{~mm}$ coplanar field of view, and spatial resolution of $0.58 \mathrm{~mm}^{2}$ at the skin's surface. In absence of anatomy-specific data, ThermaCAM ${ }^{\mathrm{TM}}$ Researcher's generic 'skin' emissivity value of 0.98 was adopted [15]. A single static TIRI was acquired from each participant. Participants were thermally imaged via the same protocol on three further occasions during their training continuum; in conjunction with scheduled medical check-up parades in weeks four, seven, and nine. Any participants presenting consistently with osseous stress injury during the course of the study were withdrawn from physical training and subject to either MRI or nuclear bone scan in accordance with Australian Army SOPs. Thermal infrared images were acquired from symptomatic subjects within the two hour period immediately prior to MRI or bone scan procedures. Subjects diagnosed with stress fracture were sent home for 6 weeks of convalescent leave.

\section{RESULTS}

Among the 474 asymptomatic thermal images obtained, were 116 complete 4-image longitudinal intrasubject TIRI sets taken over the full ten weeks of basic training. Early analysis

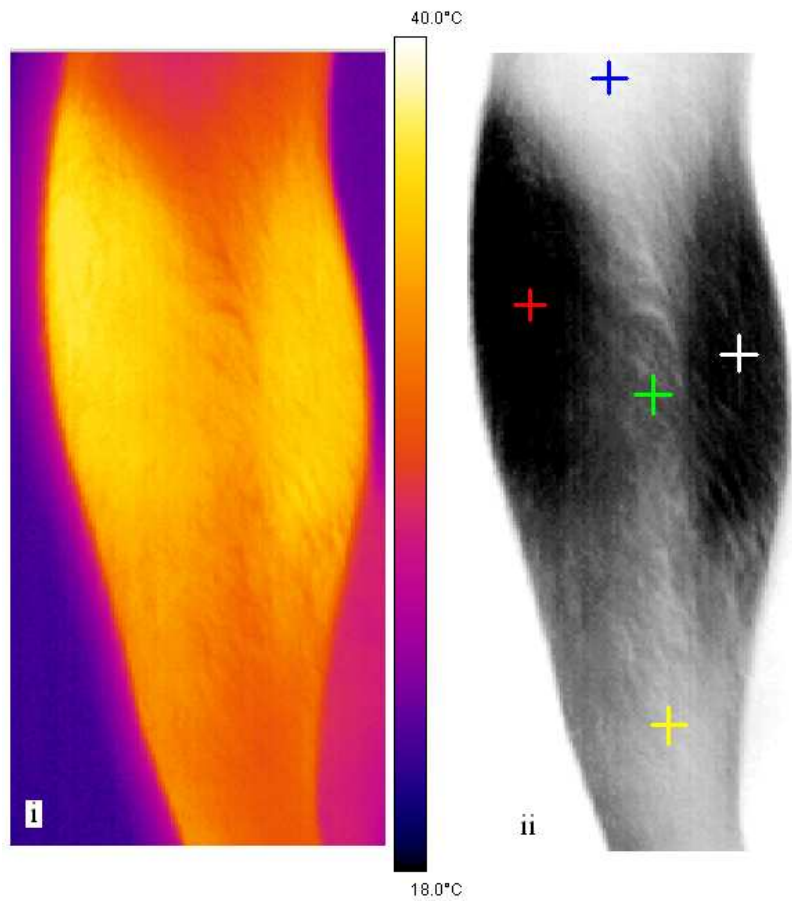

Fig. 2. TIR profile of apathological anterior lower leg; i) greybody temperature map for global emissivity of 0.98 ; ii) gray-scale negative with coloured crosshairs at geometric centers of emissively distinct regions corresponding to Thermophysiology of underlying anatomy, as explained below.

of these asymptomatic images reveals a 'normal' thermal topography for the asymptomatic anterior lower leg (Fig. 2i); the 
characteristic features of which have been validated via consideration of the thermodynamics of the underlying anatomy (Fig. 3 \& 4). As exemplified by Fig. 2, the healthy TIR profile features 5 topographically distinct regions. The blue crosshair in Fig. 2ii demarcates the point within the funnel-shaped proximal region from which emittance is typically at a minimum.

TABLE II

THERMAL CHARACTERISTICS OF APATHOLOGICAL ANTERIOR LOWER-LEG

\begin{tabular}{lcccc}
\hline \hline \multirow{2}{*}{$\begin{array}{c}\mathrm{n}=474 \\
\epsilon=0.98\end{array}$} & Mean & Mode & \multicolumn{2}{c}{ Standard Deviation } \\
\cline { 4 - 5 } $\begin{array}{l}\text { Maximum } \\
\text { Emittance / } \\
\text { Temperature* }\end{array}$ & $31.0{ }^{\circ} \mathrm{C}$ & $31.2{ }^{\circ} \mathrm{C}$ & $0.6{ }^{\circ} \mathrm{C}$ & $0.25{ }^{\circ} \mathrm{C}$ \\
$\begin{array}{l}\text { Minimum } \\
\text { Emittance / } \\
\text { Temperature* }\end{array}$ & $28.0{ }^{\circ} \mathrm{C}$ & $28.2{ }^{\circ} \mathrm{C}$ & $0.4{ }^{\circ} \mathrm{C}$ & $0.11^{\circ} \mathrm{C}$ \\
* Typical loci of maxima and minima indicated by the red and blue crosshairs \\
in Fig.2ii respectively
\end{tabular}
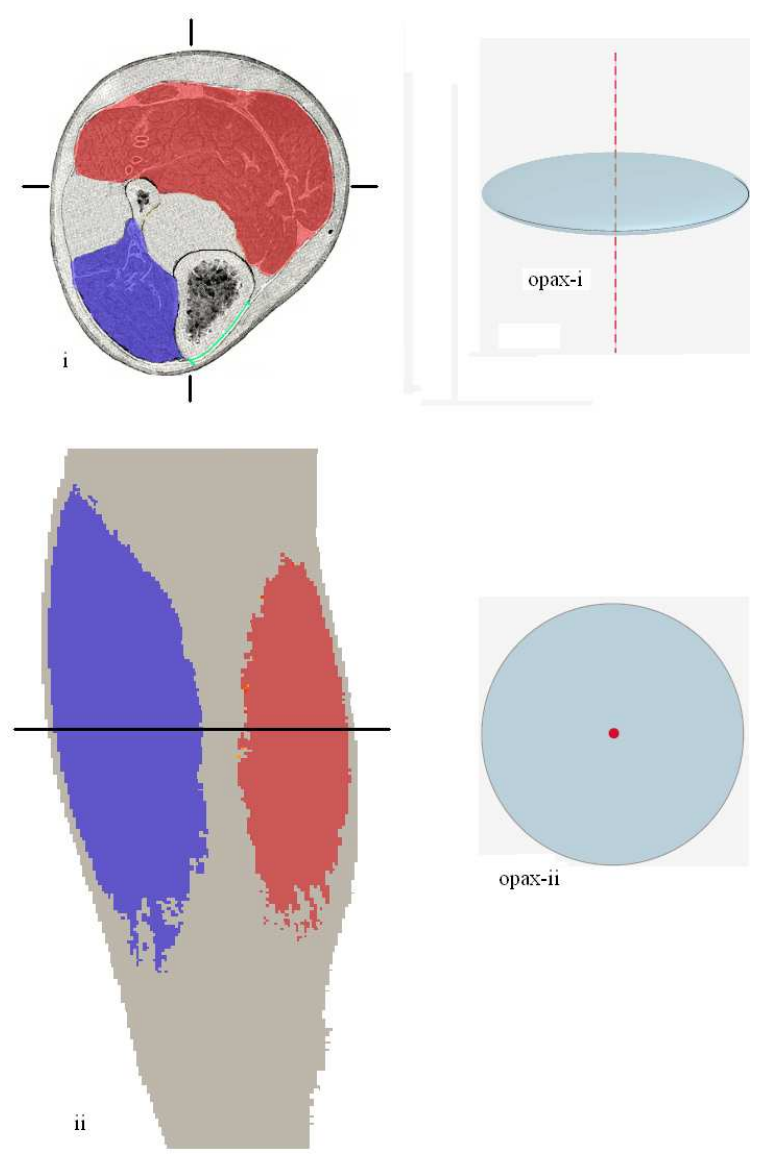

Fig. 3. Anatomic correlates of superficial thermal topography; i) axial crosssection of the lower leg showing the anterior muscle compartment (blue) the superficial posterior compartment (red), the anteromedial aspect of the tibia (green), and the relative orientation of the TIR optical axis; ii) coarsely thresheld TIR image showing discrete thermogenic regions in the archetypal anterior TIR profile of an asymptomatic lower leg.
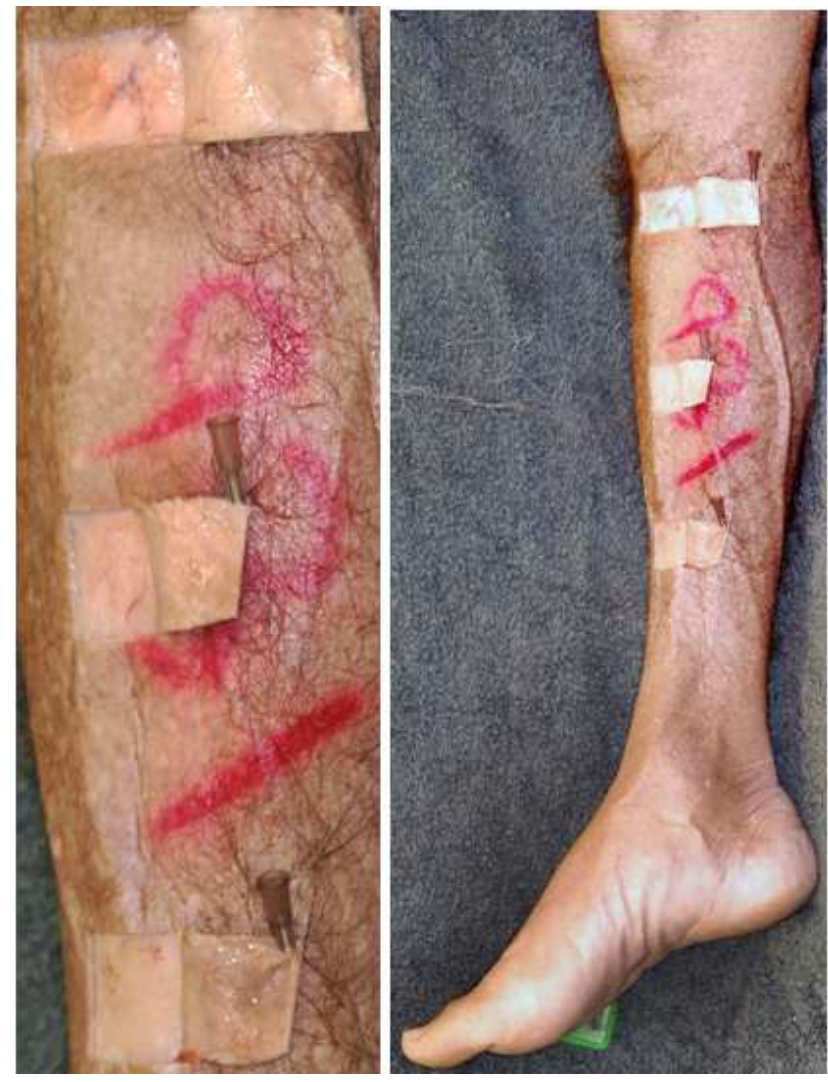

Fig. 4. Excised tissue volumes overlying anteromedial aspect of cadaveric tibia. Proximal, central, and distal excisions corresponding to figures 5, 6, and 7 respectively. Pretibial tissue volume remains avascular and heterogeneous, with thickness decreasing proximodistally.

As shown in Fig. 3i, this region overlies no thermogenic anatomy, and as shown in Fig. 5, the avascular interstitial osteocutaneous tissue volume is approximately $3.5 \mathrm{~mm}$ thick. The second row of Table 2 characterizes the average quantitative thermal characteristics of this region, as calculated from a dataset of 474 TIRI's acquired over a period of 3 months. The red crosshair in Fig 2ii demarcates the geometric centre of the point within the almond-shaped lateral region, from which emittance is typically at a maximum. As shown in blue in Fig.3, this lateral region overlies the compartment of the thermogenic tibialis anterior muscle. The white crosshair in Fig. 2ii, and the red regions in Fig. 3 correspond to the thermogenic superficial posterior muscles, with Fig. $3 \mathrm{i}$

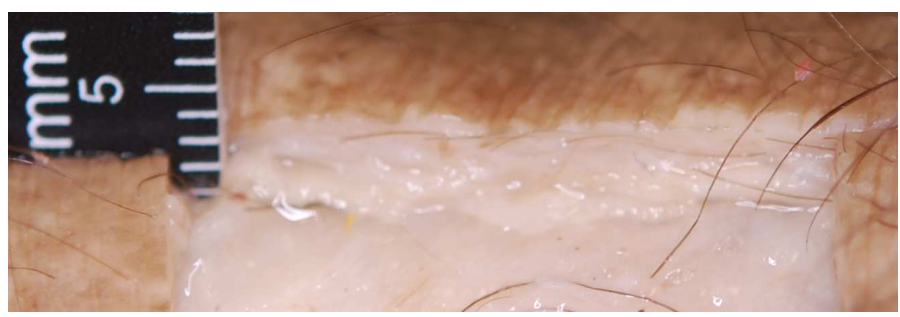

Fig. 5. Proximal excised tissue volume; corresponding to the blue crosshair in Fig. 2ii, and as shown in Fig. 4iii 


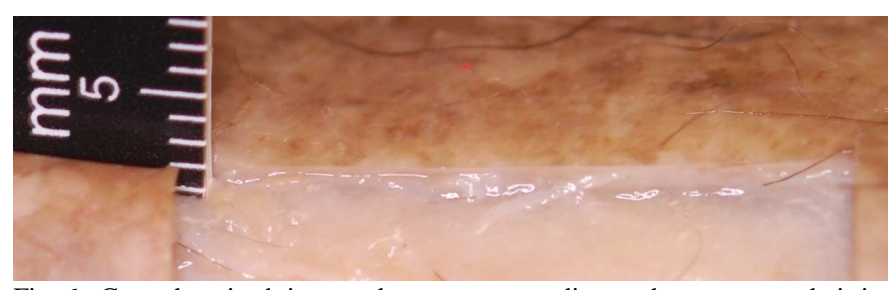

Fig. 6. Central excised tissue volume; corresponding to the green crosshair in Fig. 2ii, and as shown in Fig. 4iii

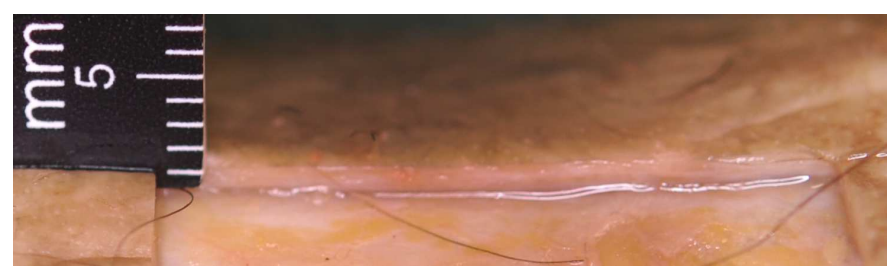

Fig. 7. Distal excised tissue volume as shown in Fig. 4iii; corresponding to the yellow crosshair in Fig. 2ii, and as shown in Fig. 4iii

illustrating the relative thickness of the overlying tissues in the direction of the optical axis, to those overlying the tibialis anterior. The green crosshair in Fig 2ii corresponds to the area overlying an avascular osteocutaneous volume of medium thickness (Fig. 6, Fig. 3i), bounded by both aforementioned thermogenic muscle compartments. The yellow crosshair of Fig. 2ii demarcates the region overlying the thinnest avascular osteocutaneous volume Fig. 7, most distal to thermogenic anatomy.

Of the 31 participants that developed symptoms consistent with osseous stress pathology, nine progressed to the point of clinical 'stress fracture' diagnosis [16] as confirmed by MRI and/or bone scan. These symptomatic results are currently being processed and analyzed for validation of perceived TIR aberration and development of a pathoanatomy-specific bioheat transfer model. Specifically, the authors are performing registration of TIRIs to; other longitudinal TIRIs to elucidate staging potential; 2D bone scintigraphic and $3 \mathrm{D}$ MR images for validation of perceived thermal aberrations, and to guide development of a pathoanatomic osteocutaneous bioheat transfer model.

\section{CONCLUSION}

A dataset of over 500 normal and symptomatic TIRI's, MRI's, and bone scan images was amassed within a controlled environment, facilitating intermodal registration and for validation of perceived pathological thermal aberrations, and to guide development of a pathoanatomic bioheat transfer model. The normal thermal topography of the asymptomatic anterior aspect of the lower leg has been defined, and validated against underlying anatomy. To the authors' knowledge, this is the first classification of lower leg emissive topography in the literature. Coarse thermographic tissue discrimination has been demonstrated, highlighting the potential of TIRI to subjectively reveal morphology.

\section{ACKNOWLEDGMENT}

The authors would like to thank Major Linda Rae, Mr. Mark Barclay, Major General Paul Alexander, Captain Michael Rhodes, Lieutenant Travis Harris, and Colonel Graeme Finney for endorsing protocols and facilitating data collection, and $\mathrm{Mr}$ John Owens and Mr Bruce Klomfass for their insight and facilitation of cadaveric dissection.

\section{REFERENCES}

[1] I. Paljak and B. Pettersson, "Thermography of Buildings," Swedish Building Research Institute, vol. Stockholm, 1972.

[2] R. Phillipson and P. Parker, "Stress Fractures," Orthopaedics and Trauma, vol. 23, pp. 137-143, 2009.

[3] D. Itskoviz, T. Marom, and I. Ostfeld, "Trends of Stress Fracture Prevalence Among Israel Defense Forces Basic Trainees " Military Medicine - Association of Military Surgeons of the U.S, vol. 76, pp. 56$59,2011$.

[4] D. Fiala, K. Lomas, and M. Stohrer, "Computer prediction of human thermoregulatory and temperature responses to a wide range of environmental conditions," International Journal of Biometeorology, vol. 45 , pp. 143-159, 2001.

[5] L. Schenkman, "Second Thoughts About CT Imaging," in Science. vol. 331 2011, pp. 1002-1004.

[6] Medicare Australia Statistics, "Category 5 Diagnostic Imaging Services," Benefit - Medicare contribution to services, vol. Financial Year July 09 - June 10, 2011.

[7] H. Hricak, D. Brenner, J. Adelstein, D. Frush, E. Hall, R. Howell, C. McCollough, F. Mettler, M. Pearce, O. Suleiman, J. Thrall, and L. Wagner, "Managing Radiation Use in Medical Imaging : A Multifaceted Challenge," Radiology, vol. 258, pp. 889-905, 2011.

[8] D. Pascoe, J. Mercer, and L. de Weerd, "Physiology of Thermal Signals," in Medical Infrared Imaging, N. Diakides and J. Bronzino, Eds. Boca Raton: CRC Press Taylor \& Francis Group, 2008, pp. 6.1 6.20 .

[9] V. Saini, S. Yadav, and S. McCormick, "Low-Intensity Pulsed Ultrasound Modulates Shear Stress Induced PGHS-2 Expression and PGE2 Synthesis in MLO-Y4 Osteocyte-Like Cells " Annals of Biomedical Engineering, vol. 39, pp. 378-393, 2011.

[10] M. Çetingül and C. Herman, "Quantification of the thermal signature of a melanoma lesion," International Journal of Thermal Sciences, vol. 50, pp. 421-431, 2010.

[11] L. De Weerd, "Free Perforator Flap Surgery and Dynamic Infrared Thermography," in Cardiovascular Research Group - Department of Medical Biology Tromso: University of Tromso, 2010, p. 53.

[12] E. Ring, H. McEvoy, A. Jung, J. Zuber, and G. Machin, "New standards for devices used for the measurement of human body temperature," Medical Engineering and Technology, vol. 34, 2010.

[13] I. International Organization for Standardization, "Basic human body measurements for technological design," ISO/TR 7250-2:2010 vol. Part 2: Statistical summaries of body measurements from individual ISO populations, 2010.

[14] FLIR Systems ${ }^{\mathrm{TM}}$, "ThermoVision ${ }^{\mathrm{TM}}$ A40V Technical Specifications," www.flirthermography.com/A40Vdata, vol. I060305PL @, 2011.

[15] FLIR, User's manual: Professional edition.Version 2.8 SR-1: 1558 071, 2004.

[16] S. P, "Discrepancies in Clinical Definitions of Stress Fractures: Implications for the United States Army," Military Medicine - ASMUS, vol. 176, pp. 60-66, 2011. 